\title{
Tuberculosis en pacientes mayores de 80 años atendidos en una institución de alta complejidad
}

\author{
Tuberculosis in patients older than 80 years at \\ a high-complexity institution
}

\author{
José Fernando García-Goez, MD. ${ }^{(1,2,4)}$; Hernán Esteban MunÉvar, EM. ${ }^{(4)}$; \\ ROBINSON PACHECO, MB. ${ }^{(2,3)}$
}

\section{Resumen}

INTRODUCCIÓN: Los mayores de 80 años son vulnerables al desarrollo de tuberculosis, debido a inmunosenescencia, comorbilidades, malnutrición y sedentarismo que predisponen a primoinfección o reactivación de infección latente. Su presentación clínica es inusual. El tratamiento antituberculoso es difícil debido a eventos adversos e interacciones farmacológicas.

OBJETIVO: describir las características clínicas, microbiológicas y los desenlaces más frecuentes en pacientes mayores de 80 años con diagnóstico de tuberculosis.

MATERIALES/MÉTODOS: estudio de cohorte retrospectivo en pacientes mayores de 80 años, con diagnóstico de tuberculosis. Del registro institucional de tuberculosis se recolectó y analizó la información clínica, microbiológica, radiológica y desenlaces al tratamiento.

RESULTADOS: entre 2011 y 2016 se diagnosticaron con tuberculosis 27 pacientes mayores de 80 años, con una mediana de edad de 84 años (RI 80-91); se identificó como factor de riesgo para esta la enfermedad pulmonar obstructiva crónica en $37 \%$, el tabaquismo $26 \%$, la diabetes $11 \%$, el cáncer $11 \%$ y $7 \%$ la infección previa por tuberculosis. El $74 \%$ tuvo retraso diagnóstico (>30 días). El 52\% presentó tuberculosis pulmonar, 26\% extrapulmonar y $22 \%$ mixta. De los pacientes con compromiso pulmonar el $41 \%$ tuvo baciloscopia positiva y $50 \%$ lesiones cavitadas en la radiografía de tórax. Luego del tratamiento de la tuberculosis se reportó curación en 37\%, muerte en $15 \%$ y pérdida del seguimiento en $37 \%$.

CONCLUSIONES: se evidencia un retraso diagnóstico dado que la presentación clínica y radiológica de la tuberculosis es inusual respecto a la población general. La forma extrapulmonar fue más frecuente en pacientes añosos. La baja tasa de curación, el alto porcentaje de mortalidad y la pérdida durante el seguimiento, representan un reto para los profesionales y sistemas de salud.

Palabras clave: adulto mayor, octogenario, tuberculosis, antituberculoso.

\begin{abstract}
INTRODUCTION: people older than 80 years are vulnerable to the development of tuberculosis; this is due to immunosenescence, comorbidities, malnutrition, and sedentariness, which predispose to primary infection or reactivation of latent infection. Its clinical presentation is unusual. Treatment of tuberculosis is difficult due to adverse events and drug interactions.
\end{abstract}

OBJECTIVE: to describe the clinical and microbiological characteristics and the most common outcomes of patients older than 80 years diagnosed with tuberculosis.

MATERIALS AND METHODS: retrospective cohort study of patients older than 80 years diagnosed with tuberculosis. Information (clinical, microbiological, radiological, and regarding outcomes and response to treatment) from the institutional tuberculosis database was collected and analyzed.

RESULTS: between 2011 and 2016, 27 patients older than 80 years were diagnosed with tuberculosis. The median age was 84 years (IR 80-91); the following risk factors for this disease were identified: chronic obstructive pulmonary disease in $37 \%$, tobacco use in $26 \%$, diabetes in $11 \%$, cancer in $11 \%$, and previous infection by tuberculosis in $7 \%$. Diagnosis was delayed $(>30$ days) in $74 \%$. The sites of infection were: $52 \%$ pulmonary, $26 \%$ extrapulmonary, and $22 \%$ mixed.

\footnotetext{
${ }^{(1)}$ Servicio de Infectologia, Fundación Valle del Lili, Cali, Colombia.

${ }^{(2)}$ Centro de Investigaciones Clínicas, Fundación Valle del Lili, Cali, Colombia.

${ }^{(3)}$ Departamento de Salud Pública, Facultad de Ciencias de la Salud, Universidad ICESI, Cali, Colombia.

${ }^{(4)}$ Estudiante de Medicina. Programa de Medicina, Facultad de Ciencias de la Salud, Universidad ICESI, Cali, Colombia. Correspondencia: José Fernando GarcíaGoez, correo electrónico: jofergarcia@ gmail.com

Recibido: 22/02/17. Aceptado: 29/02/17.
} 
Of the patients with pulmonary involvement, $41 \%$ had positive sputum examinations and $50 \%$ had lung cavitations on chest radiographs. After treatment, tuberculosis was reported to be cured in 37\%, death was reported in $15 \%$, and follow-up was lost in $37 \%$.

CONCLUSIONS: there is evidence of delayed diagnosis, due to the fact that clinical and radiological presentation of tuberculosis in this age group is unusual with regard to the general population. Extrapulmonary tuberculosis was more common in older patients. The low rates of successful treatment, the high percentage of mortality, and the loss of follow-up are a challenge for the healthcare systems and professionals.

Keywords: older patient, octogenarian, tuberculosis, antituberculous.

\section{Introducción}

La tuberculosis es una enfermedad de prevalencia mundial. En tal sentido, para 2015 la Organización mundial de la salud (OMS) reportó 10,4 millones de casos nuevos y aproximadamente 1,8 millones de muertes relacionadas, en tanto que en Colombia se notificaron 12.479 casos, con una incidencia de 31 casos por 100.000 habitantes y una tasa de mortalidad de 1.000 casos/año (1). El 40\% de los casos en Colombia se concentran en Bogotá y los departamentos de Antioquia y Valle del Cauca, con una tasa específica para este último de 40,2 casos por 100.000 habitantes $(1,2)$.

La esperanza de vida muestra un aumento significativo en el mundo; para 2050 la OMS estima que la población mayor de 80 años se incrementará cuatro veces respecto al año 2000 (3). Por su parte, el DANE (Departamento administrativo nacional de estadística) en su último censo, reporta que en Colombia la población adulto mayor se ha duplicado y el 45,19\% se concentra en los departamentos de Antioquia, Valle del Cauca y Cundinamarca, territorios nacionales donde se ubica el mayor número de casos de tuberculosis (4-6).

La población adulto mayor y en especial los mayores de 80 años, son un grupo de alto riesgo para el desarrollo de enfermedad activa por tuberculosis, dado que tienen mayores tasas de infección y peores resultados respecto al tratamiento $(3,7)$.

Dentro de los factores de riesgo se encuentran el tabaquismo, la enfermedad pulmonar obstructiva crónica (EPOC), la diabetes mellitus, la malnutrición, la insuficiencia renal crónica, el cáncer y los cambios fisiológicos del sistema inmune que acompaña el envejecimiento, conocidos como inmunosenescencia (8-11). Los principales cambios del sistema inmune del adulto mayor se asocian con la involución del timo, que se suma a una disminución de producción de linfocitos T, agotamiento de reservorio de células nativas y cambios en funciones de los neutrófilos y macrófagos, haciendo que su activación sea defectuosa $(10,12$, 13), e influya en la reactivación de la infección latente, teniendo en cuenta que la población mayor de 80 años vivió en épocas con alta prevalencia de tuberculosis $(11,12,14)$

La tuberculosis pulmonar es la forma más común en el adulto mayor de 80 años, y algunas de las presentaciones extrapulmonares son la meníngea, la ósea, la ganglionar y la del tracto gastrointestinal (14). Las manifestaciones clínicas se explicarán por cambios en la capacidad funcional y síntomas clínicos usuales como astenia, anorexia, déficit cognitivo o fiebre sin foco que fácilmente pasan inadvertidos $(8,15)$. Además, el diagnóstico de la tuberculosis es complejo debido a las presentaciones atípicas, al pobre rendimiento de los métodos diagnósticos, a las comorbilidades y a los patrones radiográficos irregulares $(8,10,14,16)$.

Adicionalmente, la información referente a la tuberculosis en el adulto mayor de 80 años en Colombia es limitada. Este estudio describe las características clínicas, los factores de riesgo y el desenlace al tratamiento antituberculoso en mayores de 80 años, atendidos en una institución de alta complejidad y referente de enfermedades infecciosas en el suroccidente de Colombia.

\section{Materiales y métodos}

Se llevó a cabo un estudio observacional, descriptivo de una cohorte histórica. Del registro institucional de tuberculosis se seleccionaron todos los pacientes mayores de 80 años, de ambos sexos, con 
diagnóstico clínico o por laboratorio a través de baciloscopia, cultivo, prueba molecular o histopatología de tuberculosis, realizados en la Fundación Valle del Lili entre 2011 y 2016. A todos los que cumplieron criterios de selección se les recolectó de los registros médicos y de laboratorio la información relacionada con variables demográficas, factores de riesgo, presentación clínica, métodos diagnósticos y resultados al tratamiento antituberculoso, información que fue consignada en una base de datos electrónica.

\section{Métodos estadísticos}

Los análisis se realizaron en el paquete estadístico Stata 12.0 (Stata Corp, College Station, TX, USA). Se hizo un análisis univariado para determinar la distribución de las variables numéricas a través de la prueba Shapiro-Wilk. Los datos se resumieron con mediana y rangos intercuartiles. Las variables cualitativas se presentaron como proporciones en tablas de frecuencia.

\section{Resultados}

Entre enero de 2011 y septiembre de 2016 se registraron 386 pacientes con diagnóstico de tuberculosis en la Fundación Valle del Lili, de los cuales $27(7 \%)$ casos correspondieron a personas mayores de 80 años, procedentes de zona urbana $(96,3 \%)$ con una mediana de edad de 84 años, RI [80-91], sin diferencias significativas por sexo. Dentro de los factores de riesgo para tuberculosis el más frecuente fue la enfermedad pulmonar obstructiva crónica con un $37 \%$, seguido de tabaquismo con $26 \%$, diabetes $11,1 \%$, cáncer $11,1 \%$, infección previa por tuberculosis $7,4 \%$ y contacto reciente con tuberculosis $7,4 \%$ (tabla 1). El 93\% de los pacientes fueron clasificados como casos nuevos de tuberculosis.

Respecto al tiempo transcurrido entre el inicio de los síntomas y el diagnóstico de tuberculosis, se presentó un retraso en el diagnóstico mayor a 30 días en el $74 \%$ de los casos. La presentación clínica más frecuente fue la pulmonar en el $51 \%$, seguida de la extrapulmonar con $26 \%$ y la mixta con $22 \%$. Las manifestaciones más comunes fueron: tos con una duración mayor a 15 días en el $63 \%$ de los pacientes, fiebre en el $52 \%$ y pérdida de peso en el $48 \%$.
Respecto al método diagnóstico, el 55\% de los pacientes con tuberculosis pulmonar incluyendo la forma mixta, tuvieron al menos una baciloscopia de esputo positiva y en el $74 \%$ de todos los pacientes el cultivo de esputo fue positivo para Mycobacterium tuberculosis; solo en un paciente se identificó un aislamiento concomitante por una micobacteria no tuberculosa. En 15 pacientes se tuvo una prueba positiva de GeneXpert MTB/RIF (12 en lavado broncoalveolar y 3 en esputo) y dos fueron resistentes a isoniacida. El patrón radiológico en los pacientes con tuberculosis pulmonar fueron lesiones pulmonares cavitadas en un $50 \%$, derrame pleural en $40 \%$ e infiltrados pulmonares en $35 \%$ (tabla 2 ).

El 70,4\% inició tratamiento antituberculoso estándar con isoniacida, rifampicina, pirazinamida y etambutol, mientras que el $29,6 \%$ recibió tratamientos individualizados debido a eventos adversos e interacciones farmacológicas, en especial con la rifampicina, o resistencia a alguno de los fármacos antituberculosos.

Del total, el 33,3\% requirió ingreso a unidad de cuidados intensivos, el resto fue manejado en hospitalización.

Para determinar los desenlaces de la cohorte, se tomó como punto de corte la finalización del estudio en septiembre de 2016. Hasta ese momento los desenlaces más frecuentes fueron: curación en $37 \%$, en tratamiento el $7,4 \%$, un paciente interrumpió el tratamiento por causas desconocidas y hubo 4 muertes correspondientes a un 14,8\% ( 3 por causas no asociadas a tuberculosis y una sí). El 37\% se perdió durante el seguimiento y no se obtuvo desenlace final.

\section{Discusión}

Este estudio es el primero en describir características clínicas, diagnóstico y desenlaces en cuanto al tratamiento de la tuberculosis en pacientes mayores de 80 años en una región con alta prevalencia, como lo es el Valle del Cauca $(2,17)$.

Los hallazgos evidencian que la prevalencia institucional de tuberculosis en pacientes mayores 
Tabla 1. Características sociodemográficas, clínicas y desenlaces de los pacientes adultos mayores de 80 años con tuberculosis $(n=27)$.

\begin{tabular}{|c|c|}
\hline & n (\%) \\
\hline Hombres & $13(48,1)$ \\
\hline Mujeres & $14(51,9)$ \\
\hline Edad (Mediana [IQ]) & $84(80-91)$ \\
\hline $\begin{array}{l}\text { Índice de masa corporal } \\
\text { (Mediana [IQ]) }\end{array}$ & $20,8(13,6-29,1)$ \\
\hline Peso insuficiente $(\mathrm{IMC}<18,4)$ & $4(14,8)$ \\
\hline Normopeso (IMC 18,5 - 24,9) & $6(22,0)$ \\
\hline Sobrepeso grado I (IMC $25-26,9)$ & $1(3,7)$ \\
\hline Sobrepeso grado II (IMC 27 - 29,9) & $2(7,4)$ \\
\hline No disponibilidad & $14(51,9)$ \\
\hline \multicolumn{2}{|l|}{ Procedencia } \\
\hline Urbana & $26(96,3)$ \\
\hline \multicolumn{2}{|l|}{ Aseguramiento } \\
\hline Contributivo & $23(85,2)$ \\
\hline Subsidiado & $4(14,8)$ \\
\hline \multicolumn{2}{|l|}{ Factores de riesgo para tuberculosis } \\
\hline $\begin{array}{l}\text { Enfermedad pulmonar obstructiva } \\
\text { crónica }\end{array}$ & $10(37)$ \\
\hline Tabaquismo & $19(26)$ \\
\hline Diabetes & $3(11,1)$ \\
\hline Cáncer & $3(11,1)$ \\
\hline Infección previa por tuberculosis & $2(7,4)$ \\
\hline Contacto reciente con tuberculosis & $2(7,4)$ \\
\hline Trasplante de órgano sólido & $0(0)$ \\
\hline Diálisis & $0(0)$ \\
\hline Hepatitis C & $0(0)$ \\
\hline VIH & $0(0)$ \\
\hline Dependencia de drogas & $0(0)$ \\
\hline Historia de prisión & $0(0)$ \\
\hline Casos nuevos de tuberculosis & $25(92,6)$ \\
\hline \multicolumn{2}{|c|}{ Tiempo con síntomas antes del diagnóstico } \\
\hline$<30$ días & $6(22,2)$ \\
\hline 31-89 días & $12(44,4)$ \\
\hline$>90$ días & $8(29,6)$ \\
\hline \multicolumn{2}{|l|}{ Presentación de la tuberculosis } \\
\hline Pulmonar & $14(51,9)$ \\
\hline Extrapulmonar & $7(25,9)$ \\
\hline Mixta & $6(22,2)$ \\
\hline
\end{tabular}

\begin{tabular}{|c|c|}
\hline & n (\%) \\
\hline \multicolumn{2}{|l|}{ Síntomas clínicos } \\
\hline Tos más de 15 días & $17(63,0)$ \\
\hline Fiebre & $14(51,9)$ \\
\hline Pérdida de peso & $13(48,1)$ \\
\hline Disnea & $9(33,3)$ \\
\hline Sudoración nocturna & $9(33,3)$ \\
\hline Dolor torácico & $3(11,1)$ \\
\hline Adenopatías & $2(7,4)$ \\
\hline Diarrea & $2(7,4)$ \\
\hline Baciloscopia de esputo positiva & $11(40,7)$ \\
\hline \multicolumn{2}{|l|}{ Hallazgos a la radiografía de tórax } \\
\hline Lesiones pulmonares cavitadas & $10(50,0)$ \\
\hline Derrame pleural & $8(40,0)$ \\
\hline Infiltrados pulmonares & $7(35,0)$ \\
\hline Granuloma & $4(20,0)$ \\
\hline Tractos fibrosos pulmonares & $2(10,0)$ \\
\hline \multicolumn{2}{|l|}{ Aislamiento de mycobacterias } \\
\hline M. Tuberculosis & $20(74,1)$ \\
\hline No identificado & $7(25,9)$ \\
\hline Coinfección con otras micobacterias & $1(3,7)$ \\
\hline \multicolumn{2}{|c|}{ Resistencia a fármacos antituberculosos } \\
\hline Ninguna & $18(66,7)$ \\
\hline Resistencia a isoniacida alto nivel & $2(7,4)$ \\
\hline $\begin{array}{l}\text { No realizada prueba de } \\
\text { susceptibilidad }\end{array}$ & $7(25,9)$ \\
\hline \multicolumn{2}{|l|}{ Inicio tratamiento estándar } \\
\hline $\mathrm{Si}$ & $19(70,4)$ \\
\hline $\begin{array}{l}\text { No (eventos adversos, } \\
\text { extrapulmonar, cavitaciones, } \\
\text { resistencia) }\end{array}$ & $8(29,6)$ \\
\hline \multicolumn{2}{|c|}{ Desenlaces del tratamiento antituberculosos } \\
\hline Éxito al tratamiento & $10(37,0)$ \\
\hline Pèrdida del seguimiento & $10(37,0)$ \\
\hline Muerte & $4(14,8)$ \\
\hline En tratamiento & $2(7,4)$ \\
\hline Interrupción & $1(3,7)$ \\
\hline No inicio tratamiento antituberculoso & $1(1,4)$ \\
\hline
\end{tabular}


Tabla 2. Principales hallazgos clínicos en pacientes adultos mayores con diagnóstico de tuberculosos según estudios relacionados.

\begin{tabular}{|c|c|c|c|c|}
\hline $\begin{array}{l}\text { Estudios } \\
\text { n (\%) }\end{array}$ & $\begin{array}{c}\text { García et al } 2016 . \\
\text { Colombia } \\
n=27\end{array}$ & $\begin{array}{c}\text { Kwon et al } \\
2012 . \\
\text { Corea } \\
n=199\end{array}$ & $\begin{array}{l}\text { Lee et al. } \\
2005 . \\
\text { corea } \\
n=119\end{array}$ & $\begin{array}{l}\text { Irache et al. } \\
2003 . \\
\text { España } \\
n=49\end{array}$ \\
\hline \multicolumn{5}{|l|}{ Factores de riesgo } \\
\hline EPOC & 10(37) & - & - & - \\
\hline Tabaquismo & $19(26)$ & $87(44)$ & - & - \\
\hline Diabetes & $3(11,1)$ & $43(22)$ & $30(25,2)$ & $7(14,3)$ \\
\hline Cáncer & $3(11,1)$ & $9(5)$ & $1(0,8)$ & $5(10,2)$ \\
\hline Infección previa por tuberculosis & $2(7,4)$ & $31(12)$ & - & $18(36,7)$ \\
\hline $\begin{array}{l}\text { Contacto reciente con } \\
\text { tuberculosis }\end{array}$ & $2(7,4)$ & - & - & - \\
\hline Trasplante de órgano sólido & $0(0)$ & - & - & - \\
\hline Diálisis & $0(0)$ & 1(1) & $2(1,7)$ & - \\
\hline Hepatitis C & $0(0)$ & - & - & - \\
\hline VIH & $0(0)$ & - & - & $0(0)$ \\
\hline Dependencia de drogas & $0(0)$ & - & - & - \\
\hline \multicolumn{5}{|l|}{ Presentación } \\
\hline Pulmonar & $14(51,9)$ & $155(78)$ & - & $37(75,5)$ \\
\hline Extrapulmonar & $7(25,9)$ & $26(13)$ & - & $12(24,5)$ \\
\hline Mixta & $6(22,2)$ & 18(9) & - & - \\
\hline \multicolumn{5}{|l|}{ Síntomas clínicos } \\
\hline Pérdida de peso & $13(48,1)$ & $5(3)$ & $46(36,1)$ & - \\
\hline Fiebre & $14(51,9)$ & $24(12)$ & $39(32,8)$ & $20(40,8)$ \\
\hline Disnea & $9(33,3)$ & $39(23)$ & $46(38,7)$ & $22(44,9)$ \\
\hline Sudoración nocturna & $9(33,3)$ & $0(0)$ & $4(3,4)$ & - \\
\hline Tos & $17(63)$ & $118(68)$ & $80(67,2)$ & $22(44,9)$ \\
\hline Dolor torácico & $3(11,1)$ & - & $5(4,2)$ & - \\
\hline Adenopatías & $2(7,4)$ & - & - & - \\
\hline Diarrea & $2(7,4)$ & - & - & - \\
\hline Baciloscopia de esputo positiva & $11(40,7)$ & $67(35)$ & $68(57,1)$ & $23(46,9)$ \\
\hline Lesiones pulmonares cavitadas & $10(50)$ & $27(16)$ & - & - \\
\hline
\end{tabular}

de 80 años es cercana al 7\%, al igual que otras cohortes evaluadas en España y Taiwán reportan prevalencias entre el 8 y el $10 \%$, siendo similar a lo esperado $(18,19)$,
Respecto al sexo, no hubo diferencia alguna, lo cual difiere de la distribución de este en la tuberculosis, en la que hay mayor prevalencia en el sexo masculino $(1,20)$. 
Con relación a las manifestaciones clínicas de los pacientes, los síntomas (tos, fiebre, sudoración nocturna y pérdida de peso) estuvieron presentes en menos de la mitad de los casos, lo que obliga al profesional de la salud a tener un alto nivel de sospecha. La literatura describe en este tipo de población manifestaciones clínicas no clásicas y suelen manifestarse como cambios en la capacidad funcional, fatiga, alteración del estado de conciencia y anorexia $(12,14)$. En este sentido, los hallazgos fueron similares a los de estudios de cohorte en España y Corea (21). Una limitante del estudio fue no poder evaluar alteraciones neurológicas dado que no fueron recolectadas.

La infección por el virus de la inmunodeficiencia humana no es un factor de riesgo para el desarrollo de tuberculosis en la población mayor de 80 años en nuestra región, como sí lo son la diabetes mellitus, el cáncer, la enfermedad pulmonar obstructiva crónica y el tabaquismo, también equivalente a los estudios de España y Corea $(12,20-22)$.

En esta cohorte el 51,9\% corresponde a tuberculosis pulmonar, en tanto que el $25,9 \%$ tuvo manifestaciones extrapulmonares como tuberculosis meníngea, gastrointestinal, ganglionar, renal, piel, articular, ósea y vertebral, igual a lo encontrado en estudios de cohorte, en los que la tuberculosis pulmonar es la forma más habitual (20-22).

En este estudio, el 92,6\% correspondió a casos nuevos de tuberculosis y el 7,4\% a casos de recaídas de la enfermedad, similar a lo evaluado en la población general (1). Se resalta que no se contó con información para determinar si los casos nuevos se trataban de reactivación de infección latente o reinfección. Existen estudios que muestran que hasta el $90 \%$ de los casos de tuberculosis en adultos mayores pueden ser causados por reactivación de infección latente $(12,14,15,20)$.

Estudios en Colombia sobre el retraso en el diagnóstico de la tuberculosis pulmonar en población general, evidencian que aproximadamente el $70 \%$ de los pacientes tenían retraso diagnóstico superior a 30 días $(17,23,24)$. En nuestro estudio se evidenció un retraso diagnóstico de la enfermedad mayor al esperado, situación que preocupa y requiere la implementación de políticas en salud con el fin de mejorar la búsqueda activa de esta entidad, en especial en la población adulto mayor. No se dispone de información para determinar si el retraso diagnóstico está asociado con condiciones sociales, clínicas del paciente o con barreras del sistema de salud.

Respecto a los desenlaces de la terapia antituberculosa, la proporción de pacientes que logró curación es del $37 \%$, resultado muy bajo respecto a las tasas de curación de la OMS mundiales y para América en el 2015 (86 y 75\% respectivamente) (1); sin embargo, el $37 \%$ de los pacientes se perdió durante el seguimiento en la institución, hecho que limitó poder realizar una evaluación adecuada.

Es preciso generar cambios en políticas de salud, con el objetivo de evaluar estrategias que garanticen una búsqueda activa de la tuberculosis, en especial en la población mayor de 80 años, y garantizar tratamientos antituberculosos mejor tolerados, con menos eventos adversos e interacciones farmacológicas, además de fortalecer los centros de atención de la población vulnerable que requiere manejo y seguimiento especial por parte de los profesionales de la salud.

\section{Conflicto de intereses}

Ninguno.

\section{Financiación}

Centro de investigaciones clínicas Fundación Valle del Lili y Universidad ICESI.

\section{Bibliografía}

1. World Health Organization. Global Tuberculosis Report. Geneva; 2016. Disponible en: http://www.who.int/tb/publications/global_report/en/ (accessed 10 july 2016)

2. Instituto nacional de salud. ¡Vivamos sin Tuberculosis!.Disponible en: http://www.ins.gov.co/Noticias/Paginas/\%C2\%A1VivamossinTuberculosis!.aspx\#.WGrVV1PhDIX (accessed 13 december 2016 ).

3. World Health Organization. "We were older then, we are younger now". Disponible en: http:/www.who.int/mediacentre/commentaries/ageing/en/ Dissiponible en: es/ageing/en/ (uctiva crponica 
4. Ministerio de la Proteccion Social, Instituto Nacional de Salud, \& Organización Panamericana de la Salud. Plan Estratégico Colombia Libre de Tuberculosis 2010-2015 Para la Expansión y Fortalecimiento de la Estrategia Alto a la TB. 2009. p. 64

5. DANE. Departamento Administrativo Nacional de Estadística. Censo General 2005. Libro Censo General. Disponible en: http://www.dane.gov.co/censo/files/libroCenso2005nacional.pdf. (Acceso 20 Ene 2015).

6. Omran AR. The epidemiologic transition: a theory of the epidemiology of population change. The Milbank Quarterly. 2005;83(4):731-57.

7. Couser JI, Glassroth J. Tuberculosis. An epidemic in older adults. Clin Chest Med. 1993;14(3):491-9.

8. Murillo C, Cabrera M, Carbonell M. Tuberculosis. Sociedad Española de Geriatría y Gerontología (SEGG). Tratado de Geriatría para residentes.

9. Pérez-Guzmán C, Vargas MH, Torres-Cruz A, Villareal Velarde H. Does aging modify pulmonary tuberculosis? A MetaAnalytical Review. Chest. 1999;116:961-7.

10. Rodríguez M, Rodríguez M. Tuberculosis en pacientes ancianos. An Med Int. 2002-,19(3):107-10.

11. Sood R. The Problem of Geriatric Tuberculosis. J Ind Acad Clin Med., 5:156-62.

12. Rajagopalan, S. Tuberculosis and aging: a global health problem. Clin Infect Dis. 2001;33:1034-9. doi:10.1086/322671

13. Romero-Cabrera AJ, Amores Hernández L, Fernández-Casteleiro E. Inmunosenescencia y fragilidad: una mirada actual. Med Int Méx. 2013;29:605-11.

14. Viloria Jiménez A, Ribera Casado JM. Criterios clínicos de sospecha de tuberculosis en pacientes de edad avanzada. Rev Clin Esp. 2002;202(7):388-90. Disponible en: http://www. revclinesp.es/es/criterios-clinicos-sospecha-tuberculosis-pacientes/articulo/13035307/. (Acceso 3 Nov 2016).
15. Fernández Fernández M, Jané Lara A, Rodríguez Vargas LE, Carreras Corzo L, García Castañeda H. Tuberculosis, comportamiento de la mortalidad en pacientes de 60 años de edad o más. Rev Cubana Med Gen Integr. 2012;28(2).

16. Recomendaciones SEPAR(Sociedad española de neumología y cirugía torácica). Normativa sobre la prevención de la tuberculosis. Grupo de trabajo del área TIR de la SEPAR. Arch Bronconeumol. 2002;38:441-51.

17. Rojas CM, Villegas SL, Piñeros HM, Chamorro EM, Durán CE, Hernández, EL. Características clínicas, epidemiológicas y microbiológicas de una cohorte de pacientes con tuberculosis pulmonar en Cali, Colombia. Biomédica. 2010;30:482-91.

18. Salvadó M, Garcia-Vidal C, Vázquez P, et al. Mortality of tuberculosis in very old people. J Am Geriatr Soc. 2010;58(1):18-22.

19. Huang-Shen L, Cheng CW, Lin MS, et al. The clinical outcomes of oldest old patients with tuberculosis treated by regimens containing rifampicin, isoniazid, and pyrazinamide. Clin Interv Aging. 2016;11:299-306.

20. López-Pelayo I, García-Martos P, Saldarreaga A, Montes De Oca M, Moreno I, González-Moya E. Características de la tuberculosis en pacientes mayores de 65 años en el área sanitaria de Cádiz (España). Rev Med Chil. 2004;132:325-30.

21. Jae HL, Dae HH, Jae WS, Hee SC. Diagnostic and therapeutic problems of pulmonary tuberculosis in elderly patients. J Korean Med Sci. 2005;20:784-9.

22. Kwon YS, Chi SY, Oh IJ, Kim KS, Kim Y, Il Lim, et al. Clinical characteristics and treatment outcomes of tuberculosis in the elderly: a case control study. BMC Infectious Diseases, 2013;13(1):1-7.

23. Cáceres-Manrique F de M, Orozco-Vargas LC. Demora en el diagnóstico de tuberculosis pulmonar en una región de Colombia. Revista de Salud Pública. 2008;10(1);94-104.

24. Rodríguez DA, Verdonck K, Bissell K, Victoria JJ, Khogali $\mathrm{M}$, Marín D, et al. Monitoring delays in diagnosis of pulmonary tuberculosis in eight cities in Colombia. Rev Panam Salud Publica. 2016;39(1). 